\title{
SOLUTIONS TO THE QUANTUM YANG-BAXTER EQUATION ARISING FROM POINTED BIALGEBRAS
}

\author{
DAVID E. RADFORD
}

\begin{abstract}
Let $R: M \otimes M \rightarrow M \otimes M$ be a solution to the quantum YangBaxter equation, where $M$ is a finite-dimensional vector space over a field $k$. We introduce a quotient $A^{\text {red }}(R)$ of the bialgebra $A(R)$ constructed by Fadeev, Reshetihkin and Takhtajan, whose characteristics seem to more faithfully reflect properties $R$ possesses as a linear operator. We characterize all $R$ such that $A^{\text {red }}(R)$ is a pointed bialgebra, and we determine all solutions $R$ to the quantum Yang-Baxter equation when $A^{\text {red }}(R)$ is pointed and $\operatorname{dim} M=2$ (with a few technical exceptions when $k$ has characteristic 2). Extensions of such solutions to the quantum plane are studied.
\end{abstract}

\section{INTRODUCTION}

In this paper we study the quantum Yang-Baxter equation from the point of view of the Faddeev, Reshetikhin and Takhtajan construction [2] to refine techniques for constructing solutions using bialgebra methods. Applying our methods in the two-dimensional case we generate with relative ease six extensive classes of solutions. In particular we find all solutions $R: M \otimes M \rightarrow M \otimes M$, where $M$ is a two-dimensional vector space over a field $k$ of characteristic not 2 , which are represented by an invertible upper triangular matrix with respect to a basis of the form $\mathscr{B}=\{m \otimes m, n \otimes m, m \otimes n, n \otimes n\}$. Included among these is the familiar quantum Yang-Baxter operator

$$
R=q\left(\sum_{i=1}^{n} e_{i i} \otimes e_{i i}\right)+\sum_{i \neq j} e_{i i} \otimes e_{j j}+\left(q-q^{-1}\right)\left(\sum_{i>j} e_{i j} \otimes e_{j i}\right)
$$

associated with quantum $S L_{q}(n)$ when $n=2$. A careful analysis of this operator when $n=2$ leads to a generalization which is described in [6, (10.8)]. We feel that our computations in the two-dimensional case point the way to the construction of families of solutions in higher dimensions.

When this paper was being revised $[4,5]$ we brought to the author's attention. These papers describe all solutions to the quantum Yang-Baxter equation (the field of definition is not clear) in the two-dimensional case based on computer analysis using Groebner basis methods. The reader should find it interesting to contrast the methods of these references and this paper.

Received by the editors January 16, 1991 and, in revised form, November 15, 1992.

1991 Mathematics Subject Classification. Primary 16W30.

Research partially supported by NSF Grant DMS-9106222. 
Our paper has its origins in Yetter's work [15] and in [12]. Let $M$ be a finitedimensional vector space over a field $k$ and suppose that $R: M \otimes M \rightarrow M \otimes M$ is a solution to the quantum Yang-Baxter equation. Let $A(R)$ be the bialgebra associated with $R$ constructed by Faddeev, Reshetikhin and Takhtajan mentioned above. Then $M$ has a left $A(R)$-module structure $(M, \cdot)$ and a right $A(R)$-comodule structure $(M, \rho)$ from which $R$ can be computed [12]. The triple $(M, \cdot, \rho)$ is a left crossed $A(R)^{\text {cop }}$-bimodule [15], and $R$ is accounted for by the prebraiding structure described in that paper. The triple $(M, \cdot, \rho)$ is also called a left quantum Yang-Baxter $A(R)$-module in [12]. Generally we will replace $A(R)$ with a more closely related bialgebra $H$. Our primary interest here is the case when $H$ is pointed. Our method of finding solutions to the quantum Yang-Baxter equation is to determine crossed bimodule structures on $M$.

Crossed bimodules arise in another context. Let $H$ be a finite-dimensional Hopf algebra over $k$. Majid shows that the left crossed $H^{\text {cop }}$-bimodules can be identified with the left modules for the Drinfel'd double (as he formulates it) of $H$ in [8]. See also [12].

This paper is organized as follows. In $\S 2$ we discuss the quantum Yang-Baxter equation $R: M \otimes M \rightarrow M \otimes M$ in connection with crossed bimodules and the bialgebra $A(R)$. We also discuss the notion of congruence, or similarity, of solutions.

We introduce $M$-reduced bialgebras in $\S 3$ and develop their properties which will be useful to us in solving the quantum Yang-Baxter equation. We describe an $M$-reduced quotient $A^{\text {red }}(R)$ of $A(R)$ and show that it satisfies a universal property similar to that satisfied by $A(R)$ [12, Theorem 2]. For us the bialgebra $A^{\text {red }}(R)$ better suited than $A(R)$ for calculation. We also find a necessary and sufficient condition for $A^{\text {red }}(R)$ to be a pointed bialgebra in terms of the operator $R$. Section 4 is devoted to a description of the quantum Yang-Baxter solutions $R$ such that $A^{\text {red }}(R)$ is generated as an algebra by its grouplike elements.

In $\S \S 5$ and 6 we treat the case when $R: M \otimes M \rightarrow M \otimes M$ is a solution to the quantum Yang-Baxter equation when $\operatorname{dim} M=2$ and $A^{\text {red }}(R)$ is pointed. We apply these results in $\S 7$ to determine those solutions $R$ in the two-dimensional case with an upper triangular matrix representation as described above. In $\S 8$ we describe a family of solutions on the quantum plane which arises from the two-dimensional case.

Very few results from the theory of coalgebras and bialgebras are needed in this paper. Sweedler's book [13] is a good reference. The reader is referred to $[12,15]$ for a discussion of crossed bimodules.

\section{Preliminaries}

Let $M$ be a finite-dimensional vector space over a field $k$ and suppose that $R=\sum_{i=1}^{n} S_{i} \otimes T_{i} \in \operatorname{End}_{k}(M) \otimes \operatorname{End}_{k}(M)$. Define $R^{12}, R^{13}, R^{23} \in \operatorname{End}_{k}(M) \otimes$ $\operatorname{End}_{k}(M) \otimes \operatorname{End}_{k}(M)$ by $R^{12}=\sum_{i=1}^{n} S_{i} \otimes T_{i} \otimes I_{M}, R^{13}=\sum_{i=1}^{n} S_{i} \otimes I_{M} \otimes T_{i}$ and $R^{23}=\sum_{i=1}^{n} I_{M} \otimes S_{i} \otimes T_{i}$. By the quantum Yang-Baxter equation we mean

$$
R^{12} \circ R^{13} \circ R^{23}=R^{23} \circ R^{13} \circ R^{12} .
$$


If we set $R^{\hat{1}}=R^{23}, R^{\hat{2}}=R^{13}$ and $R^{\hat{3}}=R^{12}$, then (1) becomes

$$
R^{\hat{3}} \circ R^{\hat{2}} \circ R^{\hat{1}}=R^{\hat{1}} \circ R^{\hat{2}} \circ R^{\hat{3}} \text {. }
$$

For finite-dimensional vector spaces $M_{1}, \ldots, M_{r}$ over $k$ we make the usual identification $\operatorname{End}_{k}\left(M_{1}\right) \otimes \cdots \otimes \operatorname{End}_{k}\left(M_{r}\right)=\operatorname{End}_{k}\left(M_{1} \otimes \cdots \otimes M_{r}\right)$ given by $T_{1} \otimes \cdots \otimes T_{r}\left(m_{1} \otimes \cdots \otimes m_{r}\right)=T_{1}\left(m_{1}\right) \otimes \cdots \otimes T_{r}\left(m_{r}\right)$, where $T_{i} \in \operatorname{End}_{k}\left(M_{i}\right)$ and $m_{i} \in M_{i}$ for $1 \leq i \leq r$. Thus we may regard $R \in \operatorname{End}_{k}(M \otimes M)$. Let $l, m, n \in M$ and write $R(m \otimes n)=\sum_{i=1}^{s} m_{i} \otimes n_{i} \in M \otimes M$. Then $R^{12}(m \otimes n \otimes l)=\sum_{i=1}^{s} m_{i} \otimes n_{i} \otimes l, R^{13}(m \otimes l \otimes n)=\sum_{i=1}^{s} m_{i} \otimes l \otimes n_{i}$ and $R^{23}(l \otimes m \otimes n)=\sum_{i=1}^{s} l \otimes m_{i} \otimes n_{i}$. With these descriptions of the operators $R^{i j}$ the quantum Yang-Baxter equation is meaningful when $M$ is any vector space over $k$.

Observe that $R^{23}=I_{M} \otimes R, R^{13}=\left(I_{M} \otimes T_{M}\right) \circ\left(R \otimes I_{M}\right) \circ\left(I_{M} \otimes T_{M}\right)$ and $R^{12}=R \otimes I_{M}$, where $T_{M}: M \otimes M \rightarrow M \otimes M$ is the "twist" map defined by $T_{M}(m \otimes n)=n \otimes m$ for $m, n \in M$. Substituting these formulations of $R^{i j}$ into (1), we see that if $R$ is an invertible solution to the quantum Yang-Baxter equation then $R^{-1}$ is as well.

Let $H$ be a bialgebra over $k$ and suppose that $M$ has a left $H$-module structure $(M, \cdot)$ and a right $H$-comodule structure $(M, \rho)$. Then $(M, \cdot, \rho)$

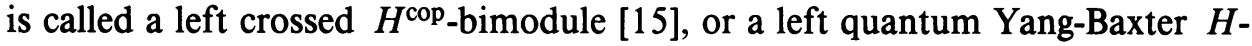
module $[6,12]$, if

$$
\sum h_{(1)} \cdot m_{\langle 1\rangle} \otimes h_{(2)} m_{(2)}=\sum\left(h_{(2)} \cdot m\right)_{\langle 1\rangle} \otimes\left(h_{(2)} \cdot m\right)_{(2)} h_{(1)}
$$

holds for all $h \in H$ and $m \in M$. Here we write $\rho(m)=\sum m_{\langle 1\rangle} \otimes m_{(2)} \in M \otimes H$ for $m \in M . H^{\text {cop }}$ is the bialgebra which is $H$ as an algebra and has coproduct $\Delta^{\mathrm{cop}}=T_{H} \circ \Delta$.

We let $H_{\mathscr{Y}} \mathscr{B}$ denote the category whose objects are those structures $(M, \cdot, \rho)$ such that (3) is satisfied and whose morphisms are simply maps of the underlying module and comodule structures. Majid shows in [8] that the category $H^{\text {cop } \mathscr{Y} \mathscr{B}}$ is naturally identified with the category of left modules for the Drinfel'd double of $H$ when $H$ is a finite-dimensional Hopf algebra (using a slightly different formulation of the double). For a variety of reasons the compatibility condition (3) is important.

To show that (3) holds for all $h \in H$ and $m \in M$ is a matter of checking that the equation holds on generators; for the set of all $h \in H$ such that (3) holds for all $m \in M$ is a subalgebra of $H$. For future reference we record:

Lemma 1. Suppose that $H$ is a bialgebra over a field $k$ and $M$ has a left $H$-module structure $(M, \cdot)$ and a right $H$-comodule structure $(M, \rho)$. Then $(M, \cdot, \rho)$ is an object of ${ }_{H} \mathscr{Y} \mathscr{B}$ if and only if (3) holds for a set of algebra generators for $H$ and a set of spanning vectors for $M$.

Suppose that $(M, \cdot, \rho)$ is an object of ${ }_{H} \mathscr{Y} \mathscr{B}$. Then $R_{(M, \cdot, \rho)}: M \otimes M \rightarrow$ $M \otimes M$ defined by $R_{(M, \cdot, \rho)}(m \otimes n)=\sum n_{(2)} \cdot m \otimes n_{\langle 1\rangle}$ for $m, n \in M$ is a solution to the quantum Yang-Baxter equation by part (b) of [12, Proposition 2]. We note that $R_{(M, \cdot, \rho)}$ is accounted for by the prebraiding structure defined in [15]. Every solution $R$ to the quantum Yang-Baxter equation arises in this manner in the finite-dimensional case, where we may take $H=A(R)$ to be the bialgebra constructed by Faddeev, Reshetikhin and Takhtajan [2]. 
Theorem 1 [12, Theorem 2]. Suppose that $M$ is a finite-dimensional vector space over a field $k$ and that $R: M \otimes M \rightarrow M \otimes M$ is a solution to the quantum YangBaxter equation. Then

(a) $M$ has the structure of an object $(M, \cdot, \rho)$ of ${ }_{A(R)} \mathscr{Y} \mathscr{B}$ such that $R=$ $R_{(M, \cdot, \rho)}$.

(b) Suppose that $H$ is a bialgebra over $k$ and that $M$ has the structure of an object $\left(M, \circ^{\prime}, \rho^{\prime}\right)$ of ${ }_{H} \mathscr{Y} \mathscr{B}$ such that $R=R_{\left(M,{ }^{\prime}, \rho^{\prime}\right)}$. Then there is a unique bialgebra map $F: A(R) \rightarrow H$ such that $\rho^{\prime}=\left(I_{M} \otimes F\right) \circ \rho$. Furthermore $a \cdot m=F(a) \bullet^{\prime} m$ for $a \in A(R)$ and $m \in M$.

The bialgebras in this paper are for the most part pointed which means that their simple subcoalgebras are one-dimensional. A one-dimensional subcoalgebra of a coalgebra $C$ over $k$ is spanned by a grouplike element, which is a nonzero $g \in C$ which satisfies $\Delta(g)=g \otimes g$, and consequently $\varepsilon(g)=1$. We let $G(C)$ denote the set of grouplike elements of $C$. By [13, Proposition 3.2.1] the set $G(C)$ is linearly independent. An element $c \in C$ is said to be nearly primitive if $\Delta(c)=g \otimes c+c \otimes g^{\prime}$ for some $g, g^{\prime} \in G(C)$. Most of the bialgebras in this paper are generated as an algebra by their nearly primitive elements.

Let $R: M \otimes M \rightarrow M \otimes M$ and $R^{\prime}: M^{\prime} \otimes M^{\prime} \rightarrow M^{\prime} \otimes M^{\prime}$ be solutions to the quantum Yang-Baxter equation, where $M$ and $M^{\prime}$ are vector spaces over $k$, and suppose that $U: M \rightarrow M^{\prime}$ is linear isomorphism. Then $R^{\prime \prime}=$ $(U \otimes U) \circ R \circ(U \otimes U)^{-1}$ is a solution as well. We say that $R$ and $R^{\prime}$ are congruent, and write $R \approx R^{\prime}$, if $R^{\prime}=R^{\prime \prime}$ for some $U$ as above. Observe that if $R$ and $R^{\prime}$ are congruent then they are similar linear transformations. When $M$ is finite-dimensional, the notion of congruence mirrors isomorphism of objects of $H \mathscr{Y} \mathscr{B B}$ by [6, (3.6.1) and (3.6.2)].

\section{3. $A^{\text {red }}(R)$ AND $M$-REDUCED BIALGEBRAS}

Let $M$ be a finite-dimensional vector space over $k$ and suppose that $R: M \otimes$ $M \rightarrow M \otimes M$ is a solution to the quantum Yang-Baxter equation. In this section we associate to $R$ a bialgebra $A^{\mathrm{red}}(R)$ which is a quotient of $A(R)$ and is in principle better suited for calculation. We will find a necessary and sufficient condition for $A^{\text {red }}(R)$ to be pointed in terms of the linear operator $R$.

Now suppose that $H$ is a bialgebra over $k,(M, \cdot, \rho)$ is an object of $H \mathscr{Y} \mathscr{B}$, and that $R=R_{(M, \cdot, \rho)}$. There are two very simple necessary and sufficient conditions for $H$ to be isomorphism to $A^{\text {red }}(R)$. The most important one of these is that $H$ be $M$-reduced.

Let $M$ be any left $H$-module. We say that $H$ is $M$-reduced if (0) is the only coideal $I$ of $H$ such that $I \cdot M=(0)$.

Proposition 1. Suppose that $H$ is a bialgebra over a field $k$ and $M$ is a left $H$ module such that $H$ is $M$-reduced. Let $\pi: H \rightarrow \operatorname{End}_{k}(M)$ be the representation of $H$ afforded by $M$. Then

(a) Suppose that $f, g: H \rightarrow H$ are coalgebra endomorphisms. If $\pi \circ f=$ $\pi \circ g$, then $f=g$.

(b) Suppose that $g \in G(H)$ and that $\pi(g)$ is invertible. If $h \in H$ and either $g h=0$ or $h g=0$, then $h=0$.

(c) Suppose that $x \in H$ is a nearly primitive. If $\pi(x)=0$, then $x=0$. 
(d) Suppose that $g, g^{\prime} \in G(H)$. If $\pi(g)=\pi\left(g^{\prime}\right)$, then $g=g^{\prime}$.

Proof. We first show part (a). Since $f$ and $g$ are coalgebra maps, $\operatorname{Im}(f-g)$ is a coideal of $H$. Since the condition $\pi \circ f=\pi \circ g$ implies that $\operatorname{Im}(f-g)$ annihilates $M$, part (a) now follows.

To show part (b), we first note that if $h \in H$ satisfies $h g=0$ or $g h=0$ then $\pi(h)=0$ since $\pi(g)$ is invertible. Now the kernel of left or right multiplication in $H$ by $g$ is a coideal of $H$ since these maps are coalgebra maps. Therefore part (b) follows.

Part (c) follows since a nearly primitive spans a coideal of $H$. Part (d) follows from part (c) since the difference of two grouplike elements of a coalgebra is a nearly primitive. This concludes the proof of the proposition.

If $H$ is a bialgebra over $k$ and $M$ is any left $H$-module, then $H$ has a quotient which is $M$-reduced. More precisely

Lemma 2. Suppose that $H$ is a bialgebra over a field $k$ and that $M$ is a left $H$-module. Let $I$ be the sum of all the coideals of $H$ which annihilate $M$. Then $I$ is a bi-ideal of $H$, and the quotient $H / I$ is an $M$-reduced bialgebra.

Proof. The sum of coideals in a coalgebra is a coideal, and the ideal generated by a coideal in a bialgebra is again a coideal. Therefore $I$ is a bi-ideal. It remains to show that $H / I$ is $M$-reduced.

Let $\pi: H \rightarrow H / I$ be the projection and suppose that $J$ is a coideal of $H / I$ which annihilates $M$. Since $\pi$ is a coalgebra map it follows that $\pi^{-1}(J)$ is a coideal of $H$. But $\pi^{-1}(J) \cdot M=(0)$, so $\pi^{-1}(J) \subseteq I$. Thus $J=(0)$, and the lemma is proved.

Primarily under the assumption that $H$ is $M$-reduced, finding solutions to the quantum Yang-Baxter equation in the finite-dimensional case and determining structures satisfying (3) are the same.

Proposition 2. Suppose that $H$ is a bialgebra over a field $k$ and that $M$ is a finite-dimensional vector space over $k$ with a left $H$-module structure $(M, \cdot)$ and a right $H$-comodule structure $(M, \rho)$. Assume that $H$ is $M$-reduced, $H$ is generated as an algebra by its smallest subcoalgebra $C$ such that $\rho(M) \subseteq M \otimes C$, and let $R=R_{(M, \cdot, \rho)}$. Then the following are equivalent:

(a) $R$ is a solution to the quantum Yang-Baxter equation.

(b) $(M, \cdot, \rho)$ is an object of $H_{H} \mathscr{B}$.

Proof. Let $I(\cdot, \rho)$ be the set of $\left(m^{*} \otimes I\right)(d(h, m))$ 's, where $m^{*}$ runs over $M^{*}$ and

$$
d(h, m)=\sum\left(h_{(1)} \cdot m_{\langle 1\rangle} \otimes h_{(2)} m_{(2)}-\left(h_{(2)} \cdot m\right)_{\langle 1\rangle} \otimes\left(h_{(2)} \cdot m\right)_{(2)} h_{(1)}\right)
$$

for $h \in H$ and $m \in M$. Then $I(\cdot, \rho)$ is a coideal of $H$ by part (a) of [12, Proposition 3]. By part (b) of the same, $R$ is a solution to the quantum YangBaxter equation if and only if $I(\cdot, \rho)$ annihilates $M$. By part (c) of the same, $(M, \cdot, \rho)$ is an object of $H \mathscr{Y} \mathscr{B}$ if and only if $I(\cdot, \rho)=(0)$. Since $H$ is $M$-reduced, the theorem follows.

Now let $M$ be a finite-dimensional vector space over $k$ and let $R: M \otimes M \rightarrow$ $M \otimes M$ be a solution to the quantum Yang-Baxter equation. Give $M$ the structure of an object of $A(R) \mathscr{Y} \mathscr{B}$ as in Theorem 1. Set $A^{\text {red }}(R)=A(R) / I(R)$, where $I(R)$ is the sum of all coideals of $A(R)$ which annihilate $M$. The 
bialgebra $A^{\text {red }}(R)$ has a universal description which is derived from that for $A(R)$.

Theorem 2. Suppose that $M$ is a finite-dimensional vector space over a field $k$ and that $R: M \otimes M \rightarrow M \otimes M$ is a solution to the quantum Yang-Baxter equation. Then

(a) $M$ has the structure of an object $(M, \cdot, \rho)$ of ${ }_{A^{\mathrm{red}}(R)} \mathscr{Y} \mathscr{B}$ such that $R=$ $R_{(M, \cdot, \rho)}$.

(b) Suppose that $H$ is a bialgebra over $k$ and that $M$ has the structure of an object $\left(M, \cdot^{\prime}, \rho^{\prime}\right)$ of $H_{H} \mathscr{Y B}$ such that $R=R_{\left(M,,^{\prime}, \rho^{\prime}\right)}$ and $H$ is $M$ reduced. Then there exists a unique map of bialgebras $F: A^{\mathrm{red}}(R) \rightarrow H$ such that $\rho^{\prime}=\left(I_{M} \otimes F\right) \circ \rho$. Furthermore, $F$ is one-one and $a \cdot m=F(a){ }^{\prime} m$ for $a \in A^{\mathrm{red}}(R)$ and $m \in M$.

Proof. Part (a) follows by Theorem 1, where $M$ is given the structures arising from the quotient $A(R) / I(R)$.

Suppose that the hypothesis of part (b) is satisfied, and regard $M$ as an object $\left(M,{ }^{\prime \prime}, \rho^{\prime \prime}\right)$ of $A(R) \mathscr{Y} \mathscr{B}$ as described in Theorem 1. By the same result there is a unique bialgebra map $G: A(R) \rightarrow H$ such that $\rho^{\prime}=\left(I_{M} \otimes G\right) \circ \rho^{\prime \prime}$. Furthermore $G$ satisfies $a{ }^{\prime \prime} m=G(a){ }^{\prime} m$ for $a \in A(R)$ and $m \in M$. The last equation implies that $G(I(R))$ annihilates $M$. Since $G$ is a coalgebra map and $I(R)$ is a coideal of $A(R)$, it follows that $G(I(R))$ is a coideal of $H$. Therefore $G(I(R))=(0)$ since $H$ is $M$-reduced. Consequently there is a bialgebra map $F: A^{\text {red }}(R) \rightarrow H$ such that $G=F \circ \pi$, where $\pi: A(R) \rightarrow A^{\text {red }}(R)$ is the projection. We conclude from the relation $G=F \circ \pi$ that $\rho^{\prime}=\left(I_{M} \otimes F\right) \circ \rho$ and $a \cdot m=F(a) \bullet^{\prime} m$ for $a \in A^{\text {red }}(R)$ and $m \in M$. This last equation shows that $\operatorname{ker} F$ is a coideal of $A^{\text {red }}(R)$ which annihilates $M$. Since $A^{\text {red }}(R)$ is $M$-reduced it follows that $F$ is one-one. Uniqueness is clear. This concludes the proof of the theorem.

By definition $A^{\text {red }}(R)$ is $M$-reduced. As a consequence of part (b) of Theorem $2, A^{\text {red }}(R)$ is generated as an algebra by its smallest subcoalgebra $C$ such that $\rho(M) \subseteq M \otimes C$. These properties characterize $A^{\text {red }}(R)$ as a bialgebra. As a corollary to the proof of the theorem:

Corollary 1. Suppose that $H$ is a bialgebra over a field $k$, that $(M, \cdot, \rho)$ is an object of $H \mathscr{Y} \mathscr{B}$, and let $R=R_{(M, \cdot, \rho)}$.

(a) Let $I$ be a bi-ideal of $H$ which annihilates $M$, and let $\left(M, \circ^{\prime}\right)$ and $\left(M, \rho^{\prime}\right)$ be the $H / I$-module structure and $H / I$-comodule structure derived from $(M, \cdot)$ and $(M, \rho)$ respectively. Then $\left(M, \cdot^{\prime}, \rho^{\prime}\right)$ is an object of ${ }_{H / I} \mathscr{Y} \mathscr{B}$ and $R=R_{\left(M, \bullet^{\prime}, \rho^{\prime}\right)}$.

(b) If $H$ is $M$-reduced and is generated as an algebra by its smallest subcoalgebra $C$ such that $\rho(M) \subseteq M \otimes C$, then $H \simeq A^{\text {red }}(R)$ as bialgebras.

Proof. To prove part (b) of the corollary, we need only note that the one-one bialgebra map $F: A^{\text {red }}(R) \rightarrow H$ of Theorem 2 is onto.

The bialgebra $A^{\mathrm{red}}(R)$ is invariant under congruence.

Corollary 2. Suppose that $M$ and $M^{\prime}$ are finite-dimensional vector spaces over a field $k$. Let $R: M \otimes M \rightarrow M \otimes M$ and $R^{\prime}: M^{\prime} \otimes M^{\prime} \rightarrow M^{\prime} \otimes M^{\prime}$ be congruent solutions to the quantum Yang-Baxter equation. Then $A^{\mathrm{red}}(R) \simeq A^{\mathrm{red}}\left(R^{\prime}\right)$ as bialgebras. 
Proof. Since $R$ and $R^{\prime}$ are congruent solutions, there is a linear isomorphism $U: M \rightarrow M^{\prime}$ such that $R^{\prime} \circ(U \otimes U)=(U \otimes U) \circ R$ by definition. Let $(M, \cdot, \rho)$ and $\left(M,{ }^{\prime}, \rho^{\prime}\right)$ be the structures on $M$ and $M^{\prime}$ respectively described in Theorem 2. Now $U$ induces a left $A^{\text {red }}(R)$-module structure $\left(M, .^{\prime \prime}\right)$ and a right $A^{\text {red }}(R)$-comodule structure $\left(M, \rho^{\prime \prime}\right)$ on $M^{\prime}$ in such a manner that $U$ is a module and comodule isomorphism. It is easy to see that $\left(M, .^{\prime \prime}, \rho^{\prime \prime}\right)$ is an object of $A_{A^{\text {red }}(R)} \mathscr{Y} \mathscr{B}$ and that $R_{\left(M^{\prime}, \bullet^{\prime}, \rho^{\prime}\right)}=R_{\left(M^{\prime}, \bullet^{\prime \prime}, \rho^{\prime \prime}\right)}$. At this point we conclude that $A^{\text {red }}(R) \simeq A^{\text {red }}\left(R^{\prime}\right)$ by part (b) of Corollary 1 .

Now we turn our attention to finding a necessary and sufficient condition for $A^{\text {red }}(R)$ to be a pointed bialgebra in terms of the linear operator $R$.

Lemma 3. Suppose that $H$ is a bialgebra over a field $k$, and let $(M, \cdot, \rho)$ be a finite-dimensional object of ${ }_{H} \mathscr{Y} \mathscr{B}$. Let $R=R_{(M, \cdot \rho)}$, suppose that $H$ is $M$-reduced and let $V$ be a subspace of $M$. Then $R(M \otimes V) \subseteq M \otimes V$ if and only if $V$ is a subcomodule of $M$.

Proof. If $L$ is a submodule of $M$ and $N$ is a subcomodule of $M$, then $R(L \otimes N) \subseteq L \otimes N$ by definition of $R=R_{(M, \cdot, \rho)}$.

Suppose that $V$ is a subspace of $M$ such that $R(M \otimes V) \subseteq M \otimes V$. Choose a basis $\left\{m_{1}, \ldots, m_{r}\right\}$ for $M$ such that $\left\{m_{1}, \ldots, m_{l}\right\}$ is a basis for $V$. Write $\rho\left(m_{j}\right)=\sum_{i=1}^{r} m_{i} \otimes h_{i j} \in M \otimes H$ for $1 \leq j \leq r$. By the comodule axioms for $(M, \rho)$ it follows that $\Delta\left(h_{i j}\right)=\sum_{l=1}^{r} h_{i l} \otimes h_{l j}$ and $\varepsilon\left(h_{i j}\right)=\delta_{i j}$ for all $1 \leq i, j \leq r$. The hypothesis $R(M \otimes V) \subseteq M \otimes V$ means that $h_{i j} \cdot M=(0)$ whenever $j \leq l<i$. But the span of these $h_{i j}$ 's is a coideal of $H$. Since $H$ is $M$-reduced, it follows that $h_{i j}=0$ whenever $j \leq l<i$. Therefore $V$ is a subcomodule of $M$, and the proof is complete.

If $V$ is an $n$-dimensional vector space, we recall that a flag for $V$ is a sequence of subspaces $(0)=V_{0} \subseteq V_{1} \subseteq V_{2} \subseteq \cdots \subseteq V_{n}=V$ such that $\operatorname{dim} V_{i}=i$ for $0 \leq i \leq n$. There is a simple characterization of when $A^{\text {red }}(R)$ is pointed in terms of flags.

Theorem 3. Suppose that $R: M \otimes M \rightarrow M \otimes M$ is a solution to the quantum Yang-Baxter equation, where $M$ is a finite-dimensional vector space over a field $k$. Regard $M$ as an object of ${ }_{A^{\text {red }}(R)} \mathscr{Y} \mathscr{B}$ as in Theorem 2. Then the following are equivalent:

(a) $A^{\text {red }}(R)$ is a pointed bialgebra.

(b) There is a flag (0)= $M_{0} \subseteq M_{1} \subseteq \cdots \subseteq M_{r}=M$ such that $R\left(M \otimes M_{i}\right) \subseteq$ $M \otimes M_{i}$ for all $0 \leq i \leq r$.

Furthermore, the subspaces in a flag satisfying the condition of part (b) are subcomodules of $M$.

Proof. We first show that part (a) implies part (b). Suppose that $A^{\text {red }}(R)$ is pointed, and let $(0)=M_{0} \subseteq M_{1} \subseteq \cdots \subseteq M_{r}=M$ be a composition series for the comodule $(M, \rho)$. Since $A^{\text {red }}(R)$ is pointed, each of the simple factors $M_{i} / M_{i-1}$ must be one-dimensional by [7, p. 354]. Therefore $\operatorname{dim} M_{i}=i$ for all $0 \leq i \leq r$, which means that the composition series is a flag. By Lemma 3 we have that $R\left(M \otimes M_{i}\right) \subseteq M \otimes M_{i}$ for all $0 \leq i \leq r$.

To show that part (b) implies part (a), suppose that $(0)=M_{0} \subseteq M_{1} \subset \cdots \subseteq$ $M_{r}=M$ is a flag such that $R\left(M \otimes M_{i}\right) \subseteq M \otimes M_{i}$ for all $0 \leq i \leq r$. Then by Lemma 3 again the flag is a composition series for the comodule $(M, \rho)$. Let 
$C$ be the smallest subspace of $A^{\text {red }}(R)$ such that $\rho(M) \subseteq M \otimes C$. Then $C$ is a subcoalgebra of $A^{\text {red }}(R)$. Regard $M$ as a left $C^{*}$-module under the (rational) action defined by $c^{*} \cdot m=\sum m_{\langle 1\rangle} c^{*}\left(m_{(2)}\right)$ for $c^{*} \in C^{*}$ and $m \in M$. Then $M$ is easily seen to be faithful $C^{*}$-module.

We have noted that $C$ generates $A^{\text {red }}(R)$ as an algebra. Thus by [3, (2.3.13) and (2.3.9)] to show that $A^{\text {red }}(R)$ is pointed it suffices to show that $C$ is pointed. For each $1 \leq i \leq r$ let $I_{i}$ be the annihilator of the one-dimensional $C^{*}$-module $M_{i} / M_{i-1}$. Then $I_{i}$ is a maximal ideal of $C^{*}$ of codimension one. Set $J=$ $I_{1} \cdots I_{r}$. Then $J \cdot M_{i} \subseteq M_{i-1}$ for all $1 \leq i \leq r$; thus $J^{r} \cdot M=(0)$. Therefore $J^{r}=(0)$ since $M$ is a faithful $C^{*}$-module. This means that $I_{1}, \ldots, I_{r}$ runs over the maximal ideals of $C^{*}$. Therefore the simple subcoalgebras of $C$ are one-dimensional by [13, Proposition 1.4.3]. Consequently $C$ is pointed, and thus part (b) impiies part (a).

\section{WHEN $A^{\text {red }}(R)$ IS GENERATED BY GROUPLIKE ELEMENTS}

Let $M$ be a finite-dimensional vector space over $k$ and suppose that $R: M \otimes$ $M \rightarrow M \otimes M$ is a solution to the quantum Yang-Baxter equation. In this section we find necessary and sufficient conditions on $R$ for $A^{\text {red }}(R)$ to be generated by grouplike elements. When $R$ is invertible and this is the case, we show how to construct $R$ from a certain family of linear automorphisms of $M$.

Suppose that $C$ is a coalgebra over $k$ and $(M, \rho)$ is a right $C$-comodule. For $g \in G(C)$ we set $M^{(g)}=\{m \in M \mid \rho(m)=m \otimes g\}$. Clearly $M^{(g)}$ is a subcomodule of $M$. Since $G(C)$ is a linearly independent set, the sum $M^{(G(C))}=\sum_{g \in G(C)} M^{(g)}$ is direct. Notice that $M^{(G(C))}$ is the sum of all onedimensional subcomodules of $M$. Now suppose that $H$ is a bialgebra over $k$ and that $M$ has the structure of an object $(M, \cdot, \rho)$ of ${ }_{H} \mathscr{Y} \mathscr{B}$. Set $R=$ $R_{(M, \cdot, \rho)}$. It is useful to note that

$$
R(x \otimes m)=g \cdot x \otimes m \quad \text { for } x \in M \text { and } m \in M^{(g)} .
$$

Consequently, if $m \in M^{(g)}$ then $R(M \otimes m) \subseteq M \otimes m$. When $H$ is $M$-reduced, this last inclusion implies that $m \in M^{(g)}$ for some $g \in G(H)$ by Lemma 3.

If a coalgebra over a field is spanned by its grouplike elements, then each of its nonzero comodules is spanned by one-dimensional subcomodules by [13, Lemma 14.0.1]. By Lemma 3 and the preceding comments we have

Corollary 3. Suppose that $M$ is a finite-dimensional vector space over a field $k$ and that $R: M \otimes M \rightarrow M \otimes M$ is a solution to the quantum Yang-Baxter equation. Then the following are equivalent:

(a) $A^{\text {red }}(R)$ is generated as an algebra by grouplike elements.

(b) $M$ is spanned by elements $m$ such that $R(M \otimes m) \subseteq M \otimes m$.

When $R$ is invertible and $A^{\text {red }}(R)$ is generated by grouplike elements, we show in Theorem 4 how to construct $R$ very simply from certain families of linear automorphisms of $M$. To do this, we need to examine the action of grouplike elements on the subspaces $M^{(g)}$.

Lemma 4. Suppose that $H$ is a bialgebra over a field $k$ and that $(M, \cdot, \rho)$ is a finite-dimensional object of ${ }_{H} \mathscr{Y} \mathscr{B}$. Set $R=R_{(M, \cdot, \rho)}$ and assume further that $R$ is an invertible linear operator and that $H$ is $M$-reduced. Let $\pi: H \rightarrow \operatorname{End}_{k}(M)$ be the representation of $H$ afforded by $(M, \cdot)$ and let $g, h \in G(H)$. Then 
(a) If $M^{(g)} \neq(0)$, then $\pi(g)$ is invertible.

(b) Suppose that $\pi(g)$ is invertible and that $M^{(h)} \neq(0)$. Then $g \cdot M^{(h)} \subseteq M^{(l)}$ for some $l \in G(H)$ which satisfies $g h=l g$.

(c) Suppose that $\pi(g)$ is invertible and $M^{(h)} \neq(0)$. Then $g \cdot M^{(h)} \subseteq M^{(g)}$ if and only if $g=h$.

Proof. We first show part (a). Choose a nonzero $m \in M^{(g)}$. Then $R(x \otimes m)=$ $\pi(g)(x) \otimes m$ for $x \in M$ by (4). Since $R$ is one-one, $\pi(g)$ therefore must be as well. Since $M$ is finite-dimensional, $\pi(g)$ must be onto since it is one-one.

To show part (b), let $m \in M^{(h)}$ be nonzero. Then $g \cdot m \neq 0$ since $\pi(g)$ is invertible. Choose a basis $g \cdot m=m_{1}, \ldots, m_{n}$ for $M$ and write $\rho(g \cdot m)=\sum_{i=1}^{n} m_{i} \otimes h_{i}$ where $h_{i} \in H$. Since $\rho(m)=m \otimes h$, the compatibility condition $\sum g_{(1)} \cdot m_{\langle 1\rangle} \otimes g_{(2)} m_{(2)}=\sum\left(g_{(1)} \cdot m\right)_{\langle 1\rangle} \otimes\left(g_{(2)} \cdot m\right)_{(2)} g_{(1)}$ translates to $g \cdot m \otimes g h=\sum_{i=1}^{n} m_{i} \otimes h_{i} g$. Therefore $h_{i} g=0$ for $1<i \leq n$. Since $H$ is $M$-reduced and $\pi(g)$ is invertible, we conclude that $h_{i}=0$ for $1<i \leq n$ by part (b) of Proposition 1. Therefore $\rho(g \cdot m)=g \cdot m \otimes h_{1}$ and $g h=\bar{h}_{1} g$. The former equation implies that $h_{1} \in G(H)$. The latter and part (b) of Proposition 1 imply that $h_{1}$ does not depend on $m$. This concludes the proof of part (b). Part (c) follows by part (b) above and part (b) of Proposition 1. This concludes the proof of the lemma.

Theorem 4. Suppose that $M$ is a nonzero finite-dimensional vector space over a field $k$ and that $R: M \otimes M \rightarrow M \otimes M$ is an invertible solution to the quantum Yang-Baxter equation. Then the following are equivalent:

(a) $A^{\text {red }}(R)$ is generated as an algebra by its grouplike elements.

(b) There is a direct sum decomposition of $M=M_{1} \oplus \cdots \oplus M_{n}$ into nonzero subspaces and a family of linear automorphisms of $T_{1}, \ldots, T_{n}$ of $M$ such that

(i) For each $i, j$ there is an $l$ such that $T_{i}\left(M_{j}\right) \subseteq M_{l}$ and $T_{i} \circ T_{j}=T_{l} \circ T_{i}$, and

(ii) $R=T_{1} \otimes E_{1}+\cdots+T_{n} \otimes E_{n}$, where $E_{i}$ is the projection of $M$ onto $M_{i}$. Proof. We first show that part (a) implies part (b). Let $g_{1}, \ldots, g_{n}$ be the list of the $g \in G(H)$ such that $M^{(g)} \neq(0)$, and set $M_{i}=M^{\left(g_{i}\right)}$. Assume that $A^{\text {red }}(R)$ is spanned by grouplike elements. Then it follows that $M=M_{1} \oplus \cdots \oplus M_{n}$ by [13, Lemma 4.0.1]. Now let $\pi: H \rightarrow \operatorname{End}_{k}(M)$ be the representation afforded by $(M, \cdot)$, and set $T_{i}=\pi\left(g_{i}\right)$. Part (b)(i) follows by part (b) of Lemma 4, and part (b)(ii) follows by (4).

The argument to show that part (b) implies part (a) is the prototype of arguments omitted in subsequent sections. Thus we will treat the details with some care.

Let $C$ be a coalgebra over $k$ with basis of grouplike elements $\left\{g_{1}, \ldots, g_{n}\right\}$. Extend the coalgebra structure of $C$ to a bialgebra structure on the tensor algebra $T(C)$ of the vector space $C$. Extend the linear map $C \rightarrow \operatorname{End}_{k}(M)$ defined by $g_{i} \mapsto T_{i}$ to an algebra homomorphism $\bar{\pi}: T(C) \rightarrow \operatorname{End}_{k}(M)$.

Suppose that the hypothesis of part (b) holds and let $I$ be the ideal of $T(C)$ generated by the differences $g_{i} g_{j}-g_{l} g_{i}$ where $1 \leq i, j \leq n$ and $l$ satisfies $T_{i} \circ T_{j}=T_{l} \circ T_{i}$. Since these differences span a coideal, it follows that $I$ is a bi-ideal. Let $H=T(C) / I$. Then $C$ is naturally embedded into $H$ as a subcoalgebra. The map $\rho: M \rightarrow M \otimes C$ defined by $\rho(m)=m \otimes g_{i}$ from $m \in$ $M_{i}$ defines a right $C$-comodule structure on $M$, and hence a right $H$-comodule structure $(M, \rho)$ on $M$. Now $\bar{\pi}$ lifts to an algebra map $\pi: H \rightarrow \operatorname{End}_{k}(M)$ 
since $\bar{\pi}(I)=(0)$. Let $(M, \cdot)$ be the left $H$-module structure determined by $\pi$.

To show that $(M, \cdot, \rho)$ is an object of $H \mathscr{Y} \mathscr{B}$, we need only verify that $\sum h_{(1)} \cdot m_{\langle 1\rangle} \otimes h_{(2)} m_{(2)}=\sum\left(h_{(2)} \cdot m\right)_{\langle 1\rangle} \otimes\left(h_{(2)} \cdot m\right)_{(2)} h_{(1)}$ holds for $h=g_{i}$ and $m \in M_{j}$ by Lemma 1 . Thus the equation we need to verify is $g_{i} \cdot m \otimes g_{i} g_{j}=$ $g_{i} \cdot m \otimes g_{l} g_{i}$. It is clear that $R_{(M, \cdot, \rho)}=T_{1} \otimes E_{1}+\cdots+T_{n} \otimes E_{n}$. Now by Lemma 2 , and parts (a) and (b) of Corollary 1, we have that part (b) implies part (a). This concludes the proof.

The reader should observe from the proof of Theorem 4 that if the hypothesis of (b)(i) is satisfied then the linear operator of part (b)(ii) is a solution to the quantum Yang-Baxter equation.

\section{SOlutions IN THE TWO-DIMENSIONAL CASE WHEN $A^{\text {red }}(R)$ IS POINTED, I}

Suppose that $M$ is a two-dimensional vector space over a field $k$. In this section and the next we are going to determine all invertible solutions $R: M \otimes$ $M \rightarrow M \otimes M$ to the quantum Yang-Baxter equation when $A^{\text {red }}(R)$ is pointed, with two technical exceptions in characteristic 2 . The pointed requirement is equivalent to $R(M \otimes m) \subseteq M \otimes m$ for some nonzero $m \in M$ by Theorem 3 . In this section we establish the basis for our general analysis and treat the case where the $m$ 's satisfying $R(M \otimes m) \subseteq M \otimes m$ span $M$.

Let $H$ be a bialgebra over $k$, let $(M, \cdot)$ be a left $H$-module structure on $M$ and let $(M, \rho)$ be a right $H$-comodule structure on $M$. Let $\pi: H \rightarrow \operatorname{End}_{k}(M)$ be the representation of $H$ afforded by $(M, \cdot)$.

Suppose that $m \in M$ is nonzero and $(M, \cdot, \rho)$ is an object of ${ }_{H} \mathscr{Y} \mathscr{B}$ such that $R=R_{(M, \cdot, \rho)}$ satisfies $R(M \otimes m) \subseteq M \otimes m$. Let $C$ be the smallest subcoalgebra of $H$ such that $\rho(M) \subseteq M \otimes C$. Then we may assume that $H$ is generated by $C$ as an algebra. By part (a) of Corollary 1 we may assume that $H$ is $M$-reduced. Thus $H \simeq A^{\text {red }}(R)$ by part (b) of the same.

By Theorem 3 we have that $\rho(m)=m \otimes A$ for some $A \in H$. Let $N=k \cdot m$ and give $M / N$ the quotient $C$-comodule structure $(M / N, \bar{\rho})$. Let $\pi: M \rightarrow$ $M / N$ be the projection and extend $\{m\}$ to a basis $\{m, n\}$ for $M$. Since $M / N$ is one-dimensional, $M / N=\operatorname{sp}(\pi(n))$ and $\bar{\rho}(\pi(n))=\pi(n) \otimes D$ for some $D \in H$. Since $\pi$ is a comodule map, it follows that $\rho(n)-n \otimes D \in \operatorname{ker}\left(\pi \otimes I_{C}\right)=$ $N \otimes C$. Therefore $B=\{m, n\}$ is a basis for $M$ such that

$$
\rho(m)=m \otimes A \quad \text { and } \quad \rho(n)=m \otimes X+n \otimes D
$$

for some $X \in C$. Applying the comodule axioms to $m$ and $n$ we see that

$$
\begin{aligned}
& A, D \text { are grouplike elements, } \\
& \Delta(X)=A \otimes X+X \otimes D \quad \text { and } \quad \varepsilon(X)=0 .
\end{aligned}
$$

The following is useful to note.

Lemma 5. Suppose that $C$ is a coalgebra over a field $k$ spanned by distinct grouplike elements $A$ and $D$ and an element $X$ satisfying $\Delta(X)=A \otimes X+$ $X \otimes D$. Then the following are equivalent:

(a) $C$ is spanned by grouplike elements.

(b) $C$ is cocommutative. 
(c) $X=\alpha(A-D)$ for some $\alpha \in k$.

(d) $C$ is spanned by $A$ and $D$.

Proof. We really need only show that part (b) implies part (c). Suppose that $C$ is cocommutative. Then $A \otimes X+X \otimes D=X \otimes A+D \otimes X$. Therefore $A, D$ and $X$ do not form a linearly independent set. Since $G(C)$ is linearly independent, it follows that $X=\alpha A+\beta D$ for some $\alpha, \beta \in k$. Using the axiom $\left(\varepsilon \otimes I_{C}\right) \circ \Delta=I_{C}$ we deduce that $\alpha+\beta=0$. This concludes our proof.

Now suppose that $(5)-(7)$ hold. Since $M^{(A)} \neq(0)$, it follows that

$$
\pi(A) \text { is invertible }
$$

by part (a) of Lemma 4. Since $R(M \otimes m) \subseteq M \otimes m$ and $R(x \otimes n)=X \cdot x \otimes$ $m+D \cdot x \otimes n$ for $x \in M$, we conclude that

$$
\pi(D) \text { is invertible }
$$

since $R$ is invertible.

For the remainder of this section we assume that $H$ is generated as an algebra (or equivalently is spanned by) its grouplike elements. Thus $C$ is spanned by grouplike elements. There are two cases to consider; $A=D$ and $A \neq D$.

Suppose that $A=D$. Now $X$ is a linear combination of grouplike elements. Since $G(C)$ is a linearly independent set and $\Delta(X)=X \otimes A+A \otimes X$, it follows that $X=0$.

Case 1. $A=D . H=k\langle A\rangle$ where

$A$ is a grouplike element .

$M$ has basis $\{m, n\}$ where

$$
\begin{gathered}
\rho(m)=m \otimes A \quad \text { and } \rho(n)=n \otimes A . \\
\pi(A)=\left(\begin{array}{ll}
a & b \\
o & c
\end{array}\right) .
\end{gathered}
$$

Regard $\mathscr{B}=\{m \otimes m, n \otimes m, m \otimes n, n \otimes n\}$ as an ordered basis for $M \otimes M$ now until the end of the next section. We frequently identify operators and their matrix representations.

Let $R=R_{(M, \cdot, \rho)}$ be a solution arising from Case 1. Then:

$$
R_{\mathscr{B}}=\left(\begin{array}{llll}
a & b & 0 & 0 \\
o & c & 0 & 0 \\
0 & 0 & a & b \\
0 & 0 & o & c
\end{array}\right)
$$

Following the outline of the proof of part (b) of Theorem 4 implies part (a), the reader can show that Case 1, as well as each of Cases 2-6 below, is realized. All that is required is the existence of a bialgebra $H^{\prime}$ over $k$ generated as an algebra by $A, D$ and $X$ which has a left $H^{\prime}$-module structure $(M, \cdot)$ and right $H^{\prime}$-comodule structure $(M, \rho)$ on $M$ satisfying the given conditions. $H^{\prime}$ need not be $M$-reduced. More relations may be needed to define the algebra structure in that case. For example, if $\pi\left(A^{n}\right)=I_{M}$, then $A^{n}-1=0$ is necessary for $H^{\prime}$ to be $M$-reduced by part (d) of Proposition 1 . In any case, $H^{\prime}$ has a unique quotient $H$ which is $M$-reduced and satisfies the given condition by part (a) of Corollary 1. By part (b) of the same $H \simeq A^{\text {red }}(R)$. Since our goal is to solve 
the quantum Yang-Baxter equation with the restriction $R(M \otimes m) \subseteq M \otimes m$, either $H$ or $H^{\prime}$ suites our purposes equally well.

Now we return to our analysis. Suppose that $A \neq D$. If $M^{(A)}=M$, then $n \otimes A=\rho(n)=m \otimes X+n \otimes D$ which implies that $A=D$. Therefore $M^{(A)}=$ $k \cdot m$. By Lemma 5 we see that $X=\alpha(A-D)$ for some $\alpha \in k$. In particular $A$ and $D$ form a basis for $C$. Since $G(C)$ is linearly independent, and $\pi(A)$ and $\pi(D)$ are invertible, $m$ is an eigenvector for both $\pi(A)$ and $\pi(D)$ by Lemma 4. Thus

$$
\pi(A)=\left(\begin{array}{ll}
a & b \\
0 & c
\end{array}\right) \text { and } \pi(D)=\left(\begin{array}{ll}
d & e \\
0 & f
\end{array}\right)
$$

Now we return to our original assumption that $(M, \cdot)$ is a left $H$-module and $(M, \rho)$ is a right $H$-comodule with no a priori connection. We are assuming that $H$ is spanned by grouplike elements $A$ and $D$. We want to find necessary and sufficient conditions for $(M, \cdot, \rho)$ to be an object of ${ }_{H} \mathscr{Y} \mathscr{B}$, given that (5)-(10) hold. These conditions are

$$
\begin{gathered}
a A X+b A D=b A^{2}+c X A \\
A D=D A \quad \text { and } \\
d D X+e D^{2}=e A D+f X D .
\end{gathered}
$$

To prove the assertion, we note by Lemma 1 that $(M, \cdot, \rho)$ is an object of $H \mathscr{Y} \mathscr{B}$ if and only if $\sum h_{(1)} \cdot x_{\langle 1\rangle} \otimes h_{(2)} x_{(2)}=\sum\left(h_{(2)} \cdot x\right)_{\langle 1\rangle} \otimes\left(h_{(2)} \cdot x_{(2)}\right)_{(2)} x_{(1)}$, which we express symbolically as

$$
(\Delta(h))(\rho(x))=\sum\left(\rho\left(h_{(2)} \cdot x\right)\right)\left(1 \otimes h_{(1)}\right),
$$

holds for algebra generators $h=A, D$ and spanning vectors $x=m, n$. Now (11)-(13) hold if and only if (14) holds for the four cases $h=A$ or $D$ and $x=m$ or $n$ collectively.

Since $X=\alpha(A-D)$ and $A D=D A,(11)$ and (13) become

$$
(\alpha a-\alpha c-b) A^{2}=(\alpha a-\alpha c-b) A D
$$

and

$$
(\alpha d-\alpha f-e) D^{2}=(\alpha d-\alpha f-e) D A
$$

respectively. Since $A \neq D$ and $H$ is $M$-reduced, it follows that $\pi(A) \neq \pi(D)$ by part (d) of Proposition 1. Therefore $\alpha a-\alpha c-b=0$ and $\alpha d-\alpha f-e=0$ by $(8)$ and $(9)$.

Case 2. $A \neq D . H=k\langle A, D, X\rangle$ where

$$
A, D \text { are grouplike elements and } \Delta(X)=A \otimes X+X \otimes D \text {. }
$$

$M$ has a basis $\{m, n\}$ where $\rho(m)=m \otimes A$ and $\rho(n)=m \otimes X+n \otimes D$.

$$
\pi(A)=\left(\begin{array}{cc}
a & \alpha(a-c) \\
0 & c
\end{array}\right), \quad \pi(D)=\left(\begin{array}{cc}
d & \alpha(d-f) \\
0 & f
\end{array}\right) \text { invertible, distinct }
$$

and

$$
\begin{gathered}
\pi(X)=\left(\begin{array}{cc}
\alpha(a-d) & \alpha^{2}(a+f-c-d) \\
0 & \alpha(c-f)
\end{array}\right), \quad \text { where } \alpha \in k \\
A D=D A, \quad X=\alpha(A-D)
\end{gathered}
$$


Let $R=R_{(M, \cdot, \rho)}$ be a solution arising from Case 2. Then

$$
R_{\mathscr{B}}=\left(\begin{array}{cccc}
a & \alpha(a-c) & \alpha(a-d) & \alpha^{2}(a+f-c-d) \\
0 & c & 0 & \alpha(c-f) \\
0 & 0 & d & \alpha(d-f) \\
0 & 0 & 0 & f
\end{array}\right)
$$

where

$$
\left(\begin{array}{cc}
a & \alpha(a-c) \\
0 & c
\end{array}\right) \neq\left(\begin{array}{cc}
d & \alpha(d-f) \\
0 & f
\end{array}\right) .
$$

In Case 1 note that $M=M^{(A)}$ and in Case 2 note that $M=M^{(A)} \oplus M^{(D)}$ is the direct sum of one-dimensional subspaces. The property $R(M \otimes u)=M \otimes u$ for all $u \in M$ is preserved under congruence. Therefore any solution arising from Case 1 cannot be congruent to any solution arising from Case 2.

6. Solutions in the two-dimensional CASE WHEN $A^{\text {red }}(R)$ Is Pointed, II

We continue the analysis of the two-dimensional case begun in the last section. Here we are going to determine the solutions to the quantum Yang-Baxter equation $R$ which satisfy the condition $R(M \otimes m) \subseteq M \otimes m$ for some nonzero $m$ unique up to scalar multiple. This is the case when $A^{\text {red }}(R)$ is pointed, but not spanned by grouplike elements.

The assumptions of $\S 5$ up to (9) are in force, and we are commencing from that point. $H$ is a bialgebra over $k$ generated by $C=\operatorname{sp}(A, D, X)$ as an algebra, $(M, \cdot)$ is a left $H$-module structure on $M$ and $(M, \rho)$ is a right $H$-comodule structure on $M$. We have a basis $B=\{m, n\}$ for $M$ such that (5)-(9) hold. We also are assuming that $H$ is $M$-reduced and is not spanned by grouplike elements.

Since $G(C)$ is linearly independent, it follows that $A$ and $D$ are the only grouplike elements of $C$. Now $M^{(A)} \neq M$ since $C$ is not spanned by grouplike elements. Therefore $M^{(A)}=k \cdot m$. Thus $m$ is an eigenvector for both $\pi(A)$ and $\pi(D)$ for the reasons cited in the preceding section. Therefore (10) holds. Since $H$ is $M$-reduced and $H$ is not generated by grouplike elements,

$$
\pi(X)=\left(\begin{array}{ll}
p & r \\
q & s
\end{array}\right) \neq 0
$$

by part (c) of Proposition 1.

Again we return to our original assumption that $(M, \cdot)$ is a left $H$-module and that $(M, \rho)$ is a right $H$-comodule with no a priori connection. We want to find necessary and sufficient conditions for $(M, \cdot, \rho)$ to be an object of $H \mathscr{Y} \mathscr{B}$, given that $(5)-(10)$ and $(15)$ hold. These conditions are

$$
\begin{gathered}
a A X+b A D=b A^{2}+c X A, \\
A D=D A, \\
d D X+e D^{2}=e A D+f X D, \\
a X^{2}+b X D+p D X+r D^{2}=r A^{2}+s X A+e A X+f X^{2}, \\
\left(\frac{p}{d}+\frac{b}{a}\right)(D-A)=0 \quad \text { and } \quad\left(\frac{s}{c}+\frac{e}{f}\right)(D-A)=0, \\
f=a \quad \text { or } \quad f=-\frac{c d}{a}, \quad \text { and } \quad q=a-\frac{c d}{a} .
\end{gathered}
$$


To prove the assertion, we recall $(14)$ that $(M, \cdot, \rho)$ is an object of $H \mathscr{Y} / \mathscr{B}$ if and only if

$$
(\Delta(h))(\rho(x))=\sum\left(\rho\left(h_{(2)} \cdot x\right)\right)\left(1 \otimes h_{(1)}\right)
$$

holds for $h=A, D, X$ and $x=m, n$. Now (16)-(18) hold if and only if (14) holds for the four cases $h=A$ or $D$ and $x=m$ or $n$ collectively. When $h=X$ and $x=m,(14)$ holds if and only if

$$
a X A+p D A=p A^{2}+q X A+d A X \text {. }
$$

When $h=X$ and $x=n,(14)$ holds if and only if (19) and

$$
c X D+q D X+s D^{2}=s D A+f D X
$$

hold. Therefore $(M, \cdot, \rho)$ is an object of $H_{H} \mathscr{B}$ if and only if (16)-(19) and (22)-(23) hold.

Assume this is the case. Then

$$
A X=\left(\frac{c}{a}\right) X A+\left(\frac{b}{a}\right)\left(A^{2}-A D\right)
$$

and

$$
A X=\left(\frac{a-q}{d}\right) X A+\left(\frac{p}{d}\right)\left(D A-A^{2}\right)
$$

are equivalent to (16) and (22) respectively. Using (17) and subtracting, we obtain the equation

$$
\left(\frac{a-q}{d}-\frac{c}{a}\right) X A+\left(\frac{p}{d}+\frac{b}{a}\right)\left(D A-A^{2}\right)=0 .
$$

Since $\pi(A)$ is invertible and $H$ is $M$-reduced,

$$
\left(\frac{a-q}{d}-\frac{c}{a}\right) X+\left(\frac{p}{d}+\frac{b}{a}\right)(D-A)=0
$$

by part (b) of Proposition 1. Since $\pi(X) \neq 0$ and $H$ is not generated by grouplike elements, we conclude that $(a-q) / d-c / a=0$, and hence $(p / d+b / a)(D-A)=0$. In a similar manner

$$
\frac{f-q}{c}-\frac{d}{f}=0 \text { and }\left(\frac{s}{c}+\frac{e}{f}\right)(D-A)=0
$$

follow. At this point the reader can easily complete the proof of our assertion.

Let us consider a natural matrix representation of a solution $R=R_{(M, \cdot, \rho)}$ which satisfies (5), (10) and (15). Since $\rho(m)=m \otimes A$ and $\rho(n)=m \otimes X+$ $n \otimes D$, we have that $R(x \otimes m)=A \cdot x \otimes m$ and $R(x \otimes n)=X \cdot x \otimes m+D \cdot x \otimes n$ for all $x \in M$. Thus

$$
\begin{aligned}
R(m \otimes m) & =a(m \otimes m), \\
R(n \otimes m) & =b(m \otimes m)+c(n \otimes m), \\
R(m \otimes n) & =p(m \otimes m)+q(n \otimes m)+d(m \otimes n) \text { and } \\
R(n \otimes n) & =r(m \otimes m)+s(n \otimes m)+e(m \otimes n)+f(n \otimes n) .
\end{aligned}
$$

The matrix of $R$ with respect to the ordered basis $\mathscr{B}$ is

$$
R_{\mathscr{B}}=\left(\begin{array}{cccc}
a & b & p & r \\
0 & c & q & s \\
0 & 0 & d & e \\
0 & 0 & 0 & f
\end{array}\right),
$$


which has the block decomposition $\left(\begin{array}{c}\pi(A) \pi(X) \\ 0\end{array} \pi(D)\right.$. For the remainder of this section we assume that $(5)-(10)$ and $(15)-(21)$ hold.

We first consider the case $A=D$, and then treat the case $A \neq D$ in three separate subcases. Most of the details are straightforward and are left to the reader.

Suppose that $A=D$. Then $q=a-c$ and either $c=a$ or $c=-c$ by (21). Assume that the characteristic of $k$ is not 2. Then $c=a$ since $c \neq 0$. Consequently $q=0$. By (16) we have that $A X=X A$. Therefore (19) reduces to $(p-s) X A=0$. Since $\pi(A)$ is invertible and $\pi(X) \neq 0$, necessarily $p-s=0$.

Case 3. $A=D$ and characteristic $k \neq 2 . H=k\langle A, X\rangle$ where

$A$ is a grouplike element and $\Delta(X)=A \otimes X+X \otimes A$.

$M$ has basis $\{m, n\}$ where $\rho(m)=m \otimes A$ and $\rho(n)=m \otimes X+n \otimes A$.

$$
\pi(A)=\left(\begin{array}{ll}
a & b \\
0 & a
\end{array}\right) \text { invertible, } \quad \pi(X)=\left(\begin{array}{ll}
p & r \\
0 & p
\end{array}\right) \neq 0
$$

and

$$
A X=X A
$$

Let $R=R_{(M, \cdot, \rho)}$ be a solution arising from Case 3. Then

$$
R_{\mathscr{B}}=\left(\begin{array}{cccc}
a & b & p & r \\
0 & a & 0 & p \\
0 & 0 & a & b \\
0 & 0 & 0 & a
\end{array}\right), \quad \text { where }\left(\begin{array}{cc}
p & r \\
0 & p
\end{array}\right) \neq 0
$$

and we assume that the characteristic of $k \neq 2$.

Observe that any bialgebra realizing Case 3 cannot be spanned grouplike elements. For if this were so, we could write $X$ as a linear combination of grouplike elements. Since $G(C)$ is linearly independent, the equation $\Delta(X)=$ $A \otimes X+X \otimes A$ would force $X=0$.

Now suppose that $A \neq D$. Since $H$ is $M$-reduced, it follows that $\pi(A) \neq$ $\pi(D)$ by part (d) of Proposition 1 . We consider the case $a \neq c$, and when $a=c$, the cases $b \neq 0$ and $b=0$.

First suppose that $a \neq c$. Then $\pi(A)$ is diagonal. We can choose $n$ to be an eigenvector of $\pi(A)$. Thus we may assume that $b=0$. We point out that this is the only time in our analysis when we place a restriction on $n$. Since $b=0$ it follows that $p=0$ by (20) and $e=0$ by (17). Since $e=0$ we have that $s=0$ by (20) again. Now (16) reduces to $a A X=c X A$. Applying $\pi$ to both sides of this equation yields $r a^{2}=r c^{2}$. Likewise (18) reduces to $d D X=f X D$, and subsequently $r d^{2}=r f^{2}$.

Case 4. $A \neq D, a \neq c . H=k\langle A, D, X\rangle$ where

$A, D$ are grouplike elements and $\Delta(X)=A \otimes X+X \otimes D$. 
$M$ has basis $\{m, n\}$ where $\rho(m)=m \otimes A$ and $\rho(n)=m \otimes X+n \otimes D$.

$$
\begin{gathered}
\pi(A)=\left(\begin{array}{ll}
a & 0 \\
0 & c
\end{array}\right), \quad \pi(D)=\left(\begin{array}{ll}
d & 0 \\
0 & f
\end{array}\right) \text { invertible, distinct, } \\
\pi(X)=\left(\begin{array}{cc}
0 & r d \\
a-\frac{c d}{a} & 0
\end{array}\right) \neq 0, \\
r a^{2}=r c^{2}, \quad r d^{2}=r f^{2}, \quad f=a \text { or } f=-\frac{c d}{a}, \\
a A X=c X A, \quad A D=D A, \quad d D X=f X D
\end{gathered}
$$

and

$$
(a-f) X^{2}=r\left(A^{2}-D^{2}\right) .
$$

By Lemma 5 any bialgebra which satisfies the conditions of Case 4 cannot be cocommutative, and hence cannot be generated by grouplike elements. For there is no $\alpha \in k$ such that $\pi(X)=\alpha(\pi(A)-\pi(D))$. Since $\pi(A X) \neq \pi(X A)$, such a bialgebra cannot be commutative either.

Let $R=R_{(M, \cdot, \rho)}$ be a solution which arises from Case 4. Then

$$
R_{\mathscr{B}}=\left(\begin{array}{cccc}
a & 0 & 0 & r \\
0 & c & a-\frac{c d}{a} & 0 \\
0 & 0 & d & 0 \\
0 & 0 & 0 & f
\end{array}\right), \quad \text { where }\left(\begin{array}{cc}
a & 0 \\
0 & c
\end{array}\right) \neq\left(\begin{array}{cc}
d & 0 \\
0 & f
\end{array}\right),
$$

$$
\left(\begin{array}{cc}
0 & r \\
a-\frac{c d}{a} & 0
\end{array}\right) \neq 0, \quad a \neq c, \quad r a^{2}=r c^{2}, \quad r d^{2}=r f^{2}, \quad f=a \text { or } f=-\frac{c d}{a} \text {. }
$$

Now suppose that $a=c$. Then $q=a-d$, and $f=a$ or $f=-d$ by (21). Assume further that $b \neq 0$. Then applying $\pi$ to (17) yields $f=d$. Applying $\pi$ to (16) gives the equation $2(a-d)=0$. Suppose that the characteristic of $k$ is not 2. Then $a=d$. Applying $\pi$ to (16) gives, $p=-b$ and $s=-e$, in conjunction with (20). Applying $\pi$ to (19) and comparing entries in the first row and second column, we find that $2 e b(e-b)=-2 r a(e-b)$. Since $\pi(A) \neq \pi(D)$ it follows that $e \neq b$. Note that (19) can be simplified using (16)-(18).

Case 5. $A \neq D, a=c, b \neq 0$ and characteristic $k \neq 2 . H=k\langle A, D, X\rangle$ where

$$
A, D \text { are grouplike elements and } \Delta(X)=A \otimes X+X \otimes D \text {. }
$$

$M$ has basis $\{m, n\}$ where $\rho(m)=m \otimes A$ and $\rho(n)=m \otimes X+n \otimes D$.

$$
\begin{gathered}
\pi(A)=\left(\begin{array}{ll}
a & b \\
0 & a
\end{array}\right), \quad \pi(D)=\left(\begin{array}{ll}
a & e \\
0 & a
\end{array}\right) \text { invertible, } e \neq b, \\
\pi(X)=\left(\begin{array}{cc}
-b & -\frac{e b}{a} \\
0 & -e
\end{array}\right),
\end{gathered}
$$

$A X-X A=\frac{b}{a}\left(A^{2}-A D\right), \quad A D=D A, \quad$ and $\quad D X-X D=\frac{e}{a}\left(A D-D^{2}\right)$.

Since $\pi(X)=\alpha(\pi(A)-\pi(D))$ has no solution for $\alpha \in k$, it follows that any bialgebra which satisfies Case 5 is not cocommutative, and hence is not 
generated by grouplike elements, by Lemma 5 . Also note that $\pi(A D) \neq \pi\left(A^{2}\right)$. Therefore such a bialgebra is not commutative.

Suppose that $R=R_{(M, \cdot, \rho)}$ is a solution which arises from Case 5. Then

$$
R_{\mathscr{B}}=\left(\begin{array}{cccc}
a & b & -b & -\frac{e b}{a} \\
0 & a & 0 & -e \\
0 & 0 & a & e \\
0 & 0 & 0 & a
\end{array}\right), \quad \text { where } e \neq b, b \neq 0
$$

and we assume the characteristic of $k$ is not 2 .

Now suppose that $b=0$. Then $A$ is central by (16), $p=0$ and $f s=-e a$ by (20). Applying $\pi$ to (18) and comparing entries in the first row and second column, we conclude by (18) that $r a^{2}=r d^{2}$ when $f=a$. Observe that (19) reduces to $(a-f) X^{2}=(s+e) A X+r\left(A^{2}-D^{2}\right)$ since $A$ and $X$ commute. Applying $\pi$ to both sides of this equation and comparing entries in the first row and second column again, we see that $r e(a+d)=0$ when $f=a$.

Let $\alpha \in k$. Since $H$ is $M$-reduced, $X-\alpha(A-D)=0$ if and only if $\pi(X-\alpha(A-D))=0$ by part (c) of Proposition 1. Thus by Lemma 5 we see that $\pi(X)=\alpha(\pi(A)-\pi(D))$ is not possible, since $H$ is not generated by grouplike elements. This equation has a solution for $\alpha$ if and only if $a=d$, $f=-d$ and $2 a r=-e^{2}$.

Case 6. $A \neq D, a=c, b=0 . H=k\langle A, D, X\rangle$ where

$$
A, D \text { are grouplike elements and } \Delta(X)=A \otimes X+X \otimes D \text {. }
$$

$M$ has basis $\{m, n\}$ where $\rho(m)=m \otimes A$ and $\rho(n)=m \otimes X+n \otimes D$.

$$
\begin{array}{r}
\pi(A)=\left(\begin{array}{ll}
a & 0 \\
0 & a
\end{array}\right), \quad \pi(D)=\left(\begin{array}{ll}
d & e \\
0 & f
\end{array}\right) \text { invertible, distinct, } \\
\pi(X)=\left(\begin{array}{cc}
0 & \stackrel{r}{a e} \\
a-d & -\frac{a}{f}
\end{array}\right), \\
f=a \text { or } f=-d .
\end{array}
$$

(If $f=a$, then $r a^{2}=r d^{2}$ and $r e(a+d)=0$; if $f=-d$, then $a \neq d$ or $2 a r \neq-e^{2}$.)

$$
A X=X A, \quad A D=D A, \quad d D X=e D^{2}=e A D+f X D
$$

and

$$
(a-f) X^{2}=r\left(D^{2}-A^{2}\right)+\left(e-\frac{a e}{f}\right) A X .
$$

By Lemma 5 any bialgebra satisfying Case 6 is not cocommutative, and hence is not generated by grouplike elements. Since $\pi(X D) \neq \pi(D X)$ it follows that any such bialgebra is not commutative. 
Let $R=R_{(M, \cdot, \rho)}$ be a solution which arises from Case 6. Then (C6) $R_{\mathscr{B}}=\left(\begin{array}{cccc}a & 0 & 0 & \stackrel{r}{a e} \\ 0 & a & a-d & -\frac{a}{f} \\ 0 & 0 & d & e \\ 0 & 0 & 0 & f\end{array}\right)$

$$
\text { where }\left(\begin{array}{ll}
a & 0 \\
0 & a
\end{array}\right) \neq\left(\begin{array}{ll}
d & e \\
0 & f
\end{array}\right),\left(\begin{array}{cc}
0 & \stackrel{r}{a e} \\
a-d & -\frac{1}{f}
\end{array}\right) \neq 0,
$$

and $f=a$ (in which case $r a^{2}=r d^{2}$ and $r e(a+d)=0$ ) or $f=-d$ (in which case $a \neq-d$ or $\left.2 a r \neq-e^{2}\right)$.

\section{UPPER TRIANGULAR SOLUTIONS IN THE TWO-DIMENSIONAL CASE}

Our investigations in $\S \S 5$ and 6 give us a complete determination of the invertible upper triangular solutions in the two-dimensional case when the characteristic of $k$ is not 2 .

Theorem 5. Suppose that $R$ is an invertible upper triangular $4 \times 4$ matrix with coefficients in a field $k$ of characteristic not 2 . Write $R=(\mathscr{A} \mathscr{\mathscr { L }})$, where $\mathscr{A}$, $\mathscr{X}, \mathscr{O}$ and $\mathscr{D}$ are $2 \times 2$ matrices. Then $R$ is a solution to the quantum Yang-Baxter equation if and only if it has one of the following forms:

$$
R=\left(\begin{array}{llll}
a & b & 0 & 0 \\
0 & c & 0 & 0 \\
0 & 0 & a & b \\
0 & 0 & 0 & c
\end{array}\right)
$$

$$
R=\left(\begin{array}{cccc}
a & \alpha(a-c) & \alpha(a-d) & \alpha^{2}(a+f-c-d) \\
0 & c & 0 & \alpha(c-f) \\
0 & 0 & d & \alpha(d-f) \\
0 & 0 & 0 & f
\end{array}\right), \quad \text { where } \mathscr{A} \neq \mathscr{D}
$$

$$
R=\left(\begin{array}{cccc}
a & b & p & r \\
0 & a & 0 & p \\
0 & 0 & a & b \\
0 & 0 & 0 & a
\end{array}\right), \quad \text { where } \mathscr{Z} \neq 0
$$

(C4) $R$ is congruent to a matrix of the type

$$
\left(\begin{array}{cccc}
a & 0 & 0 & r \\
0 & c & a-\frac{c d}{a} & 0 \\
0 & 0 & d & 0 \\
0 & 0 & 0 & f
\end{array}\right)
$$

where $\mathscr{A} \neq \mathscr{D}, \mathscr{X} \neq 0$ and $a \neq c, r a^{2}=r c^{2}, r d^{2}=r f^{2}, f=a$ or $f=-c d / a$. 


$$
\begin{gathered}
R=\left(\begin{array}{cccc}
a & b & -b & -e b / a \\
0 & a & 0 & -e \\
0 & 0 & a & e \\
0 & 0 & 0 & a
\end{array}\right), \quad \text { where } e \neq b, b \neq 0 \\
R=\left(\begin{array}{cccc}
a & 0 & 0 & r \\
0 & a & a-d & -a e / f \\
0 & 0 & d & e \\
0 & 0 & 0 & f
\end{array}\right)
\end{gathered}
$$

where $\mathscr{A} \neq \mathscr{D}, \mathscr{Z} \neq 0$ and $f=a$ (in which case $r a^{2}=r d^{2}$ and $r e(a+d)=0$ ) or $f=-d$ (in which case $a \neq d$ or $2 a r \neq-e^{2}$ ).

Proof. Choose a basis $\{m, n\}$ for a two-dimensional vector space $M$ over $k$, and let $\mathscr{B}=\{m \otimes m, n \otimes m, m \otimes n, n \otimes n\}$ be regarded as an ordered basis for $M \otimes M$. The proof follows from the calculations of the previous two sections, where we identify endomorphisms of $M \otimes M$ and their matrices with respect to $\mathscr{B}$.

Now we turn to the problem of congruence. Suppose that $R, R^{\prime}: M \otimes$ $M \rightarrow M \otimes M$ are solutions to the quantum Yang-Baxter equation which arise from Cases 3-6. Let $B^{\prime}=\left\{m^{\prime}, n^{\prime}\right\}$ be an ordered basis for $M$ and regard $\mathscr{B}=\{m \otimes m, n \otimes m, m \otimes n, n \otimes n\}$ as an ordered basis for $M \otimes M$. Let $B^{\prime}=\left\{m^{\prime}, n^{\prime}\right\}$ be another ordered basis for $M$ and $\mathscr{B}^{\prime}$ be the counterpart of $\mathscr{B}$. Now $R=R_{(M, \cdot, \rho)}$, where $(M, \cdot, \rho)$ is an object of ${ }_{H} \mathscr{Y} \mathscr{B}$ for some bialgebra $H$ over $k$. We will assume that $R(M \otimes m)=M \otimes m$ and that $H$ is generated as an algebra by $A, D$ and $X$ where (5)-(10) and (15) are satisfied. Following the notation of $\S 6$ we write

$$
R_{\mathscr{B}}=\left(\begin{array}{llll}
a & b & p & r \\
0 & c & q & s \\
0 & 0 & d & e \\
0 & 0 & 0 & f
\end{array}\right) .
$$

Likewise $R^{\prime}=R_{\left(M,,^{\prime}, \rho^{\prime}\right)}$, where $\left(M, .^{\prime}, \rho^{\prime}\right)$ is an object of $H^{\prime} \mathscr{Y O B}$ for some bialgebra $H^{\prime}$ over $k$. We assume that $R^{\prime}\left(M \otimes m^{\prime}\right)=M \otimes m^{\prime}$, and write $m^{\prime}$ for $m, n^{\prime}$ for $n, A^{\prime}$ for $A$, and $\ldots a^{\prime}$ for $a \ldots$ Let $B^{\prime}=\left\{m^{\prime}, n^{\prime}\right\}$ and $\mathscr{B}^{\prime}=\left\{m^{\prime} \otimes m^{\prime}, n^{\prime} \otimes m^{\prime}, m^{\prime} \otimes n^{\prime}, n^{\prime} \otimes n^{\prime}\right\}$ denote the corresponding bases for $M$ and $M \otimes M$ respectively. Then we have

$$
R_{\mathscr{B ^ { \prime }}}^{\prime}=\left(\begin{array}{cccc}
a^{\prime} & b^{\prime} & p^{\prime} & r^{\prime} \\
0 & c^{\prime} & q^{\prime} & s^{\prime} \\
0 & 0 & d^{\prime} & e^{\prime} \\
0 & 0 & 0 & f^{\prime}
\end{array}\right) .
$$

For an $m \times n$ matrix $A=\left(a_{i j}\right)$ and an $r \times s$ matrix $B=\left(b_{k l}\right)$ we let $A \odot B$ denote the $m r \times n s$ matrix which has the block form

$$
\left(\begin{array}{ccc}
A b_{11} & \cdots & A b_{1 s} \\
\vdots & & \vdots \\
A b_{r 1} & \vdots & A b_{r s}
\end{array}\right) .
$$

As a first step towards showing that Cases 1-6 are fundamentally different we establish 
Lemma 6. Suppose that $M$ is a two-dimensional vector space over a field $k$ and that $R, R^{\prime}: M \otimes M \rightarrow M \otimes M$ are solutions to the quantum Yang-Baxter equation which arise from Cases 3-6. Assume that $R \approx R^{\prime}$, and let $B$ and $B^{\prime}$ be bases for $M$ such that (29) and (30) are satisfied respectively. Then

(a) There is an invertible $2 \times 2$ upper triangular matrix $A$ with coefficients in $k$ such that $R_{\mathscr{B}^{\prime}}^{\prime}(A \odot A)=(A \odot A) R_{\mathscr{B}}$. In particular $A \odot A$ is upper triangular.

(b) The diagonals of $R_{\mathscr{B}}$ and $R_{\mathscr{B}}^{\prime}$, are identical.

(c) $q=q^{\prime}$.

Proof. Let $U: M \rightarrow M$ be a linear automorphism such that $R^{\prime} \circ(U \otimes U)=$ $(U \otimes U) \circ R$, and let $x \in M$. Then $R(M \otimes x)=M \otimes x$ if and only if $R^{\prime}(M \otimes U(x))=M \otimes U(x)$. Thus it follows that the property $R(M \otimes x)=M \otimes x$ for some $x \in M$, unique up to scalar multiple, is invariant under congruence. Therefore $U(m)=\alpha m^{\prime}$ for some $\alpha \in k$. In particular $R^{\prime}$ arises from Cases 3-6 also and $A=[U]_{B}^{B^{\prime}}$ is upper triangular. By change of basis calculations it follows that $R^{\prime}(A \odot A)=(A \odot A) R$. We have established part (a), from which parts (b) and (c) follow.

Using Lemma 6 we can show that two solutions arising from different cases among Cases 1-6 are not congruent.

Proposition 3. Let $M$ be a two-dimensional vector space over a field $k$ and suppose that $R, R^{\prime}: M \otimes M \rightarrow M \otimes M$ are solutions to the quantum YangBaxter equation which arise from Cases 1-6. If $R \approx R^{\prime}$ then they arise from the same case.

Proof. Suppose that $R \approx R^{\prime}$. Then $A^{\text {red }}(R) \simeq A^{\text {red }}\left(R^{\prime}\right)$ as bialgebras by Corollary 2. $R_{\mathscr{B}}$ and $R_{\mathscr{G}}^{\prime}$, have the same diagonal by part (a) of Lemma 6 when $R$ and $R^{\prime}$ arise from Cases 3-6.

Suppose that $R$ arises from Cases 1 or 2 . Then $A^{\text {red }}(R)$ is spanned by grouplike elements. This is not the case if $R^{\prime}$ arises from Cases 3-6. Therefore $R^{\prime}$ arises from Cases 1 or 2 . In this situation we showed that $R$ and $R^{\prime}$ arise from the same case in $\S 5$.

Suppose that $R$ arises from one of Cases 3-6. If $R$ arises from Case 3, then $A^{\text {red }}(R)$ is cocommutative, which is not true if $R$ arises from a subsequent case. By part (a) of Lemma 6, $\pi(A)$ and $\pi\left(A^{\prime}\right)$ are similar matrices. With these facts in mind, a quick inspection shows that $R^{\prime}$ cannot arise from a case subsequent to the one in which $R$ is found. Hence the proposition follows.

Using Lemma 6 it is not hard to determine congruence classes of solutions arising from the same case.

Let $R: M \otimes M \rightarrow M \otimes M$ be a solution to the quantum Yang-Baxter equation. Then $R^{\circ}=T_{M} \circ R \circ T_{M}$ is also. Let $m \in M$. Then $R(M \otimes m) \subseteq M \otimes m$ if and only if $R^{\circ}(m \otimes M) \subseteq m \otimes M$. Now suppose that the characteristic of $k$ is not 2. Then Theorem 5 can be used to describe invertible solutions $R$ when $\operatorname{dim} M=2$ which satisfy the condition $R(m \otimes M) \subseteq m \otimes M$ for some nonzero $m \in M$. Now assume that $k$ is algebraically closed. Then by the results of $[6, \S 7]$ the remaining solutions to determine when $M$ is two-dimensional are those for which $M$ is a simple left $A^{\text {red }}(R)$-module and a simple right $A^{\text {red }}(R)$ comodule, where $M$ is given the structure of an object of ${ }_{A^{\text {rod }}(R)} \mathscr{Y} \mathscr{B}$ according to Theorem 2. 


\section{SOlutions IN THE TWO-DIMENSIONAL CASE WHICH EXTEND TO THE QUANTUM PLANE}

We determine the two-dimensional objects of ${ }_{H} \mathscr{Y} \mathscr{B}$ in the setting of $\S 6$ which make the quantum plane an object of $H \mathscr{Y} / \mathscr{B}$ as described below. By part (b) of Proposition 4 below these structures are accounted for by Case 4 .

The homogeneous components of the quantum plane basically account for all irreducible $\mathscr{U}_{q}\left(s l_{2}\right)$-modules [6]. These components are subobjects of the quantum plane as we regard it as an object of ${ }_{H} \mathscr{Y} \mathscr{B}$.

Let $H$ be a bialgebra over a field $k$, and suppose that $A$ is a $k$-algebra which has a left $H$-module structure $(A, \cdot)$. Then $(A, \cdot)$ is said to be a left $H$-module algebra if

$$
h \cdot(a b)=\sum\left(h_{(1)} \cdot a\right)\left(h_{(2)} \cdot b\right) \quad \text { and } \quad h \cdot 1=\varepsilon(h) 1
$$

for all $h \in H$ and $a, b \in A$. Now assume that $A$ is an algebra over $k$ which has a right $H$-comodule structure $(A, \rho)$. Then $(A, \rho)$ is said to be a right $H$-comodule algebra if

$$
\rho(a b)=\rho(a) \rho(b) \quad \text { and } \quad \rho(1)=1 \otimes 1
$$

for $a, b \in A$. Now suppose that $(M, \cdot)$ is a left $H$-module. Then there is a unique extension of the module structure on $M$ to a module structure $(T(M), \cdot M)$ on the tensor algebra $T(M)$ of the vector space $M$ which results in a left $H$-module algebra structure on $T(M)$. Likewise there is a unique extension of a right $H$-comodule structure $(M, \rho)$ on $M$ which gives $T(M)$ the structure of a right $H^{\mathrm{op}}$-comodule algebra $\left(T(M), \rho_{M}\right) . H^{\mathrm{op}}$ is the bialgebra which is $H$ as a coalgebra and whose multiplication is given by $m^{\text {op }}=m \circ T_{H}$. If $(M, \cdot, \rho)$ is an object of $H \mathscr{Y} \mathscr{B}$, then by $[6,(4.3 .3)]$ it follows that $\left(T(M), \cdot_{T}, \rho_{T}\right)$ is an object of ${ }_{H} \mathscr{Y} \mathscr{B}$.

Let $k\{x, y\}$ be the free $k$-algebra on the symbols $x$ and $y$, in other words the tensor algebra of the vector space over $k$ with basis $\{x, y\}$. For $\mathbf{q} \in k$ we let $k[x, y]_{\mathbf{q}}$ denote the algebra quotient $k\{x, y\} /(y x-\mathbf{q} x y)$. When $\mathbf{q}$ is not zero and not a root of unity $k[x, y]_{\mathbf{q}}$ is referred to as the quantum plane.

Proposition 4. Suppose that $H$ is a bialgebra over a field $k$ and that $(M, \cdot, \rho)$ is an object of $H \mathscr{Y G O B}$. Assume $\{m, n\}$ is a basis for $M$ and that (5)-(10) and (15) are satisfied. Regard $T(M)$ as the object $\left(T(M),{ }^{\top}, \rho_{T}\right)$ of ${ }_{H} \mathscr{Y} \mathscr{B}$. Suppose further that $H$ is $M$-reduced and is not generated by grouplike elements. Let $\{x, y\}$ be a basis of $M$ also, and assume that $\mathbf{q} \in \mathbf{k}$ is not zero. Then

(a) Suppose that $\mathbf{q}=1$. Then $y x-\mathbf{q} x y$ spans a one-dimensional subcomodule of $T(M)$ if and only if $A X=X A$.

(b) Suppose that $\mathbf{q} \neq 1$ and one of Cases 3-6 is satisfied. Then $y x-\mathbf{q} x y$ spans a one-dimensional subcomodule and submodule of $T(M)$ if and only if

(i) $x=\alpha m, y=\beta^{\prime} n$ for some $\alpha, \beta^{\prime} \in k$ (in which case $\mathbf{q}=\frac{c}{a}$ ) or

(ii) $x=\beta n, y=\alpha^{\prime} m$ for some $\alpha^{\prime}, \beta \in k$ (in which case $\mathbf{q}=\frac{a}{c}$ ), and

(iii) the conditions of Case 4 hold and $q=0$, or $q \neq 0, f=a$ and $r=0$. If the conditions of (a) or (b) hold, then $k[x, y]_{\mathbf{q}}=T(M) /(y x-\mathbf{q} x y)$ is an object of $H \mathscr{Y} \mathscr{B}$ with the quotient structures.

Proof. Write $x=\alpha m+\beta n$ and $y=\alpha^{\prime} m+\beta^{\prime} n$ where $\alpha, \beta, \alpha^{\prime}, \beta^{\prime} \in k$. Since $\{x, y\}$ is a basis for $M$ it follows that $\alpha \beta^{\prime}-\alpha^{\prime} \beta \neq 0$. Set $\mathbf{a}=\alpha \beta^{\prime}-\mathbf{q} \alpha^{\prime} \beta$, $\mathbf{b}=\alpha \alpha^{\prime}(1-\mathbf{q}), \mathbf{c}=-\left(\alpha^{\prime} \beta-\mathbf{q} \alpha \beta^{\prime}\right)$ and $\mathbf{d}=\beta \beta^{\prime}(1-\mathbf{q})$. 
Suppose that $y x-\mathbf{q} x y$ generates a one-dimensional subcomodule of $T(M)$, which is to say that $\rho_{T}(y x-\mathbf{q} x y)=(y x-\mathbf{q} x y) \otimes C$ for some $C \in H$. This happens if and only if

$$
\begin{gathered}
\mathbf{a} A X+\mathbf{b} A^{2}+\mathbf{d} X^{2}=\mathbf{c} X A+\mathbf{b} C \\
\mathbf{d} D X=\mathbf{c}(D A-C), \\
\mathbf{d} X D=\mathbf{a}(C-D A) \text { and } \\
\mathbf{d} D^{2}=\mathbf{d} C .
\end{gathered}
$$

Suppose that $\mathbf{q}=1$. Then $\mathbf{b}=\mathbf{d}=0$ and $\mathbf{a}=\mathbf{c} \neq 0$. Therefore (31)-(34) hold if and only if $C=A D$ and $A X=X A$.

Now suppose that $\mathbf{q} \neq 1$. We first assert that $\mathbf{d}=0$. If $D^{2} \neq C$ then $\mathbf{d}=0$ by (34). If $D^{2}=C$, then $\mathbf{d} X=\mathbf{a}(D-A)$ follows from (33) and part (b) of Proposition 1 since $H$ is $M$-reduced. But by assumption $H$ is not generated by grouplike elements. Therefore $\mathbf{d}=0$ in this case as well.

Since $\mathbf{d}=0$ and $\mathbf{q} \neq 1$, it follows that $\beta \beta^{\prime}=0$. Since $\alpha \beta^{\prime}-\alpha^{\prime} \beta \neq 0$, we easily see at this point that $\mathbf{a} \neq 0, \mathbf{c} \neq 0$ and $\mathbf{a} \neq \mathbf{c}$. In particular, $C=A D$, and (31)-(34) come down to

$$
C=A D \quad \text { and } \quad \mathbf{a} A X+\mathbf{b} A^{2}=\mathbf{b} D A+\mathbf{c} X A .
$$

We now solve for $A X$ in (16), which is $a A X+b A D=b A^{2}+c X A$, and substitute into the last equation to obtain $\left(\mathbf{a}\left(\frac{c}{a}\right)-\mathbf{c}\right) X A=\left(\mathbf{b}+\mathbf{a}\left(\frac{b}{a}\right)\right)\left(D A-A^{2}\right)$. Again, since $H$ is $M$-reduced and not generated by grouplike elements, we have the $\mathbf{a}\left(\frac{c}{a}\right)-\mathbf{c}=0$. We have shown that $y x-\mathbf{q} x y$ generates a one-dimensional subcomodule of $T(M)$ if and only if

$$
\beta \beta^{\prime}=0, \quad \mathbf{a} c=\mathbf{c} a \quad \text { and } \quad(\mathbf{b} a+\mathbf{a} b)(D-A)=0 .
$$

Since $\mathbf{a} \neq \mathbf{c}$ it follows that $a \neq c$. Therefore the conditions of Case 4 must be satisfied. In particular $b=0$ and $D \neq A$. Therefore $\mathbf{b}=0$ by (35). This means $\alpha \alpha^{\prime}=0$. Since $\alpha \beta^{\prime}-\alpha^{\prime} \beta \neq 0$, either $x=\alpha m$ and $y=\beta^{\prime} n$, or $x=\beta n$ and $y=\alpha^{\prime} m$. In the first case $\mathbf{q}=\frac{c}{a}$, and in the second $\mathbf{q}=\frac{a}{c}$ by (35). In either case it is easy to see that $X \cdot_{T}(y x-\mathbf{q} x y)=\alpha(y x-\mathbf{q} x y)$ for some $\alpha \in k$ if and only if $q(a-f)=0=r(c-d)$, from which the conditions of part (b)(iii) follow. We have established the forward implications in parts (a) and (b). At this point the reader is left with the small exercise of finishing the arguments for the reverse implications. This concludes our proof.

When $\mathbf{q}=-1$, there are families of 2-dimensional objects in $H_{\mathcal{Y}} \mathscr{B}$ which turn the exterior algebra of $M$ into an object of ${ }_{H} \mathscr{Y} \mathscr{B}$. When $\mathbf{q}=1$ there are families of such objects which turn the symmetric algebra of $M$ into an object of $H \mathscr{Y} \mathscr{B}$. These are not hard to determine.

Let $m=y$ and $n=x$. Then the conditions of part (b) of Proposition 4 are met in the special case of (C4),

$$
R=\left(\begin{array}{cccc}
q b & 0 & 0 & 0 \\
0 & b & q b-q^{-1} c & 0 \\
0 & 0 & c & 0 \\
0 & 0 & 0 & q b
\end{array}\right) \quad \text { where } b, c \neq 0 \text { and } q \neq 1,0, q^{2} \neq \frac{c}{b}
$$


This example is discussed in depth in $[6, \S 10]$. Observe that

$$
\begin{aligned}
& R(y \otimes y)=q b(y \otimes y), \\
& R(x \otimes y)=b(x \otimes y), \\
& R(y \otimes x)=c(y \otimes x)+\left(q b-q^{-1} c\right)(x \otimes y), \\
& R(x \otimes x)=q b(x \otimes x) .
\end{aligned}
$$

This particular solution suggests the quantum Yang-Baxter operator [6, (10.8)]

$$
\begin{aligned}
R= & q b\left(\sum_{i=1}^{n} e_{i i} \otimes e_{i i}\right)+b\left(\sum_{i>j} e_{i i} \otimes e_{j j}\right) \\
& +c\left(\sum_{i<j} e_{i i} \otimes e_{j j}\right)+\left(q b-q^{-1} c\right)\left(\sum_{i>j} e_{i j} \otimes e_{j i}\right),
\end{aligned}
$$

which is a generalization of the familiar

$$
R=q\left(\sum_{i=1}^{n} e_{i i} \otimes e_{i i}\right)+\sum_{i \neq j} e_{i i} \otimes e_{j j}+\left(q-q^{-1}\right)\left(\sum_{i>j} e_{i j} \otimes e_{j i}\right)
$$

The reader is referred to $[6, \S 10]$ for details. In particular the extension of the solution described above to the quantum plane is computed there.

\section{REFERENCES}

1. V. G. Drinfel'd, Quantum groups, Proc. Internat. Congr. Math., Berkeley, Calif., 1987, pp. 798-820.

2. L. D. Faddeev, N. Yu Reshetikhin, and L. A. Takhtajan, Quantization of Lie groups and Lie algebras. I, II, LOMI preprints, 1987.

3. R. L. Heyneman and D. E. Radford, Reflexitivity and coalgebras of finite type, J. Algebra 28 (1974), 215-246.

4. J. Hietarinta, All solutions to the constant quantum Yang-Baxter equation in two dimensions, Phys. Lett. A 165 (1992), 245-251.

5. __ Solving the two-dimensional constant quantum Yang-Baxter equation, preprint, 1992.

6. L. Lambe and D. E. Radford, Algebraic aspects of the quantum Yang-Baxter equations, J. Algebra 154 (1993), 228-289.

7. R. G. Larson, Characters of Hopf algebras, J. Algebra 17 (1971), 352-368.

8. S. Majid, Doubles of quasitriangular Hopf algebras, Comm. Algebra 19 (1991), 3061-3073.

9. __ Physics for algebraists: non-commutative and non-cocommutative Hopf algebras by a bicrossproduct construction, J. Algebra 129 (1990), 1-91.

10. $\_$Quasitriangular Hopf algebras and Yang-Baxter equations, Internat. J. Modern Phys. A 5 (1990), 1-91.

11. D. E. Radford, Minimal quasitriangular Hopf algebras, J. Algebra 157 (1993), 285-315.

12. $\ldots$ Solutions to the quantum Yang-Baxter equation and the Drinfel'd double, J. Algebra 161 (1993), 20-32.

13. M. E. Sweedler, Hopf algebras, Math. Lecture Notes Ser., Benjamin, New York, 1969.

14. P. Smith, Quantum groups for ring theorists, preprint.

15. D. N. Yetter, Quantum groups and representations of monodial categories, Math. Proc. Cambridge Philos. Soc. 108 (1990), 261-290.

Department of Mathematics, Statistics, and Computer Science, University of Illinois at Chicago, Chicago, Illinois 60680

E-mail address: radford@math.uic.edu 